\title{
Distance-dependent electron transfer rate of immobilized redox proteins: A statistical physics approach
}

\author{
Sören Georg, ${ }^{1}$ Julia Kabuss, ${ }^{1}$ Inez M. Weidinger, ${ }^{2}$ Daniel H. Murgida, ${ }^{3}$ Peter Hildebrandt, ${ }^{2}$ Andreas Knorr, ${ }^{1}$ and \\ Marten Richter ${ }^{1, *}$ \\ ${ }^{1}$ Institut für Theoretische Physik, Nichtlineare Optik und Quantenelektronik, Technische Universität Berlin, \\ Hardenbergstraße 36, 10623 Berlin, Germany \\ ${ }^{2}$ Max-Volmer-Laboratorium für Biophysikalische Chemie, Institut für Chemie, Technische Universität Berlin, \\ Straße des 17, Juni 135, 10623 Berlin, Germany \\ ${ }^{3}$ Departamento de Química Inorgánica, Analítica y Química Física /INQUIMAE-CONICET, Facultad de Ciencias Exactas y Naturales, \\ Universidad de Buenos Aires, Ciudad Universitaria, Pab. 2, Piso 1, C1428EHA Buenos Aires, Argentina
}

(Received 6 November 2009; revised manuscript received 18 February 2010; published 1 April 2010)

\begin{abstract}
The electron transfer kinetics of redox proteins adsorbed on metal electrodes coated with self-assembled monolayers (SAMs) of mercaptanes shows an unusual distance-dependence. For thick SAMs, the experimentally measured electron transfer rate constant $k_{\text {exp }}$ obeys the behavior predicted by Marcus theory [R. A. Marcus and N. Sutin, Biochim. Biophys. Acta 811, 265 (1985)], whereas for thin SAMs, $k_{\text {exp }}$ remains virtually constant [Z. Q. Feng et al., J. Chem. Soc., Faraday Trans. 93, 1367 (1997)]. In this work, we present a simple theoretical model system for the redox protein cytochrome c electrostatically bound to a SAM-coated electrode. A statistical average of the electron tunneling rate is calculated by accounting for all possible orientations of the model protein. This approach, which takes into account the electric field dependent orientational distribution, allows for a satisfactory description of the "saturation" regime in the high electric field limit. It further predicts a nonexponential behavior of the average electron transfer processes that may be experimentally checked by extending kinetic experiments to shorter sampling times, i.e., $\ll 1 / k_{\text {exp }}$. For a comprehensive description of the overall kinetics in the saturation regime at sampling times of the order of $1 / k_{\exp }$, it is essential to consider the dynamics of protein reorientation, which is not implemented in the present model.
\end{abstract}

PACS number(s): 82.20.-w, 87.14.et, 87.10.-e, 73.40.Jn

\section{INTRODUCTION}

Numerous experimental studies have focused on the analysis of the distance-dependence of the electron transfer (ET) processes between the redox center of metalloproteins and metal electrodes [1-7]. Particularly detailed electrochemical and spectroscopic investigations have been carried out with the heme protein cytochrome c (Cyt-c), which readily adsorbs electrostatically to metal electrodes coated with self-assembled monolayers (SAMs) of carboxylterminated alkanethiols $[1,2,4,6,8-10]$ (Fig. 1). SAM-coated electrodes allow for a precise variation of the ET distance via altering the number of methylene groups of the alkanethiol. For long alkanethiols with more than 10 methylene groups, the measured ET rate increases exponentially with decreasing distance, indicating electron tunneling to be the ratelimiting step, in accordance with the predictions by Marcus theory (see Sec. I A). For shorter alkanethiols, however, the experimental rate constant is virtually distance-independent, pointing to a process other than electron tunneling to be the rate-limiting step. This process was recently identified as a reorientation or rotational diffusion of the immobilized protein [10]. On the other hand, recent experiments have shown, that there is a similar unusual distance-dependence of the short range ET, involving the related cytochrome $b_{562}$, where no protein reorientation could be detected [12]. Our calculations will show, that protein reorientation is not necessarily

\footnotetext{
*marten.richter@tu-berlin.de
}

the only cause for the observed "plateau" region of the ET for short SAM lengths. Already the statistical distribution of orientations for short distances to the electrode leads to the deviation from the exponential behavior of the ET rate within length depending time windows of the experiment. Inspection of the protein surface reveals several positively charged lysine residues that may serve for electrostatic binding to the negatively charged SAM surface [13]. Thus, different SAM-protein complexes can be formed. In each of these complexes, the redox site, i.e., the heme group, adopts a different orientation relative to the metal surface, associated with different electronic couplings and thus different electron tunneling rates $[14,15]$. As a consequence, the experimentally determined ET rates reflect the interplay between the orientational distribution, the orientation-

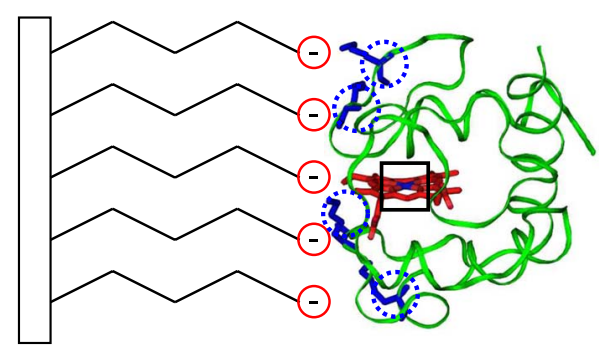

FIG. 1. (Color online) Illustration of the electrostatic binding of Cyt-c via four surface lysines [indicated by blue (black) dotted circles] on carboxylate headgroups (sketch of Cyt-c taken from VMD [11]). The redox center of the protein (Fe-ion) is located in the center of the heme plane (indicated by black squares). 
dependent electron tunneling, and the transition between different SAM-protein complexes, i.e., protein reorientation. Both, the orientational distribution and the reorientation rate are governed by the strength of the electrostatic interactions between the protein and the SAM, which depends on the specific lysine residues involved and on the interfacial electric field $[14,16]$. With decreasing SAM thickness, the electric field strength at the protein binding site increases, which is expected to slow down protein reorientation such that it becomes rate-limiting for SAMs of short alkanethiols [10]. In this way, the distance-dependence of the experimentally determined ET rate can be qualitatively understood. However, a quantitative description that can account for all experimental data is not yet available and, in view of the complexity of the interfacial processes, quite a challenge. In this work, we have employed the methodology of statistical physics to analyze the effect of the orientational distribution of the protein on the ET kinetics. The approach is applied to a simple model system for the protein-SAM complex as a first step toward a comprehensive and consistent description of the redox process of Cyt-c and other redox proteins on electrodes.

This paper is organized as follows. First, we give a brief overview of the Marcus theory of electron transfer and the statistical method to calculate an average ET rate. Second, the model system for Cyt-c on a SAM-coated electrode is introduced and the potential energy is calculated, which involves a derivation of the electrostatic potential. Third, the average rate constant is evaluated, by taking into account the orientational degrees of freedom of the model protein. Finally, we discuss the overpotential [17] dependence of the ET rate for various SAM lengths.

\section{A. Marcus theory of electron transfer}

The ET rate constant for nonadiabatic electron transfer, that is, for weak donor-acceptor interaction $\left(V_{D A}\right)$ between the donor $(D)$ and the acceptor $(A)$, is given by the Marcus approach $[18,19]$

$$
k_{E T}=\left|V_{D A}\right|^{2} \sqrt{\frac{\pi}{\hbar^{2} \lambda k_{B} T}} e^{-\left(\Delta G^{0}+\lambda\right)^{2} / 4 \lambda k_{B} T},
$$

where $\Delta G^{0}$ corresponds to the driving force of the ET reaction and $\lambda$ is the reorganization energy. $k_{B} T$ is the thermal energy, given by the temperature $T$.

For heterogeneous ET reactions, in which the metal electrode serves as a reaction partner, Eq. (1) must be modified to take account of the continuum of electronic states in the electrode. This can be achieved by averaging Eq. (1) over the Fermi-Dirac distribution [20]. Assuming a constant density of states $\rho$ and approximating the Fermi-Dirac distribution by a step function, one obtains [21]

$$
k_{E T}=\left|V_{D A}\right|^{2} \frac{\pi}{\hbar} \rho\left(1-\operatorname{erf}\left(\frac{e_{0} \eta+\lambda}{\sqrt{4 \lambda k_{B} T}}\right)\right),
$$

where the driving force is expressed in terms of the overpotential $\eta$. $e_{0}$ and "erf" denote the elementary charge and the Gaussian error function, respectively.

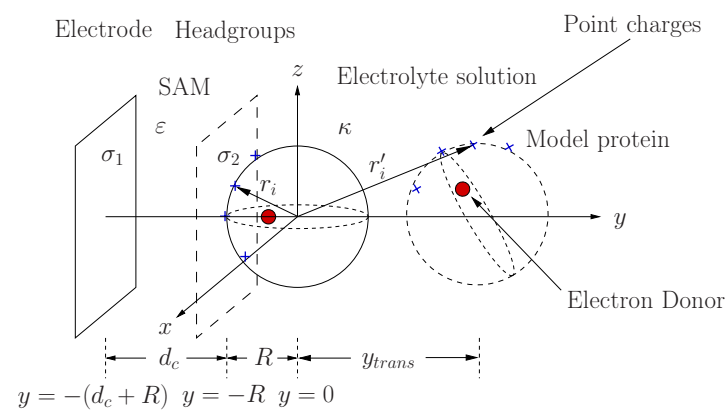

FIG. 2. (Color online) Model system for Cyt-c on a SAM-coated electrode. The second sphere in dashed lines is shown to illustrate the translation of Cyt-c with respect to the SAM and the rotation in the global coordinate system. $r_{i}$ and $r_{i}^{\prime}$ are the vectors of a point charge on the sphere in its initial position and after the translational and rotational motion, respectively. $R$ denotes the radius of the sphere, $d_{c}$ the SAM length. $\sigma_{1}$ and $\sigma_{2}$ are the surface charge densities on the electrode and the SAM, $\varepsilon$ is the dielectric constant of the SAM and $\kappa$ the inverse Debye length of the electrolyte solution.

$V_{D A}$ is the donor-acceptor coupling matrix element and can be assumed as $[18,22]$

$$
V_{D A}=V_{D A}^{0} e^{-\left(d-d_{0}\right) \cdot \xi / 2},
$$

where $\xi$ is the effective tunneling decay parameter. $V_{D A}^{0}$ is the coupling element at a distance $d_{0}$. Equations (2) and (3) nicely describe the experimental data for the ET rates at thick SAM coatings, but fail for thin SAM coatings [2,9,23-25].

\section{B. Statistical average of the tunnelling rate}

In order to calculate an average ET rate, which takes into account the ensemble of different protein-SAM complexes, we apply a standard method from classical statistical physics.

In order to calculate the ensemble average of the electron transfer rate $k_{E T}$, we introduce a simple model system and calculate the Hamilton function for the ET system, which is given as the sum of the kinetic $\left(T_{k i n}\right)$ and electrostatic potential energy $(W)$. The kinetic energy depends on the generalized coordinates and momenta, whereas the potential energy only depends on the coordinates. Since the tunneling rate is independent of the momenta, the ensemble average of $k_{E T}$ is given by

$$
\left\langle k_{E T}\right\rangle=\frac{\int d \boldsymbol{s} \exp \left(-\frac{W(s)}{k_{B} T}\right) k_{E T}(s)}{\int d s \exp \left(-\frac{W(s)}{k_{B} T}\right)},
$$

such that it is sufficient to derive an expression for the potential energy $W(s)$.

\section{MODEL SYSTEM}

The model system, illustrated in Fig. 2, is kept as simple as possible to mimic only those forces and properties that are considered to be essential for the interfacial electron transfer process of Cyt-c to be described, i.e., the asymmetric distri- 
bution of charge residues on the protein surface and the resultant electrostatic interactions with the charged SAMcoated electrode. Hence, the model does not claim to represent an approximate description of the threedimensional structure and charge distribution of the protein and the protein/SAM/metal interfaces, which instead requires molecular dynamics simulations [14]. Correspondingly, we have used a sphere with radius $\mathrm{R}$ as a model for the protein, with four positive point charges $\left(q_{i}=e_{0}>0\right)$ asymmetrically distributed on the surface of the sphere in analogy to the positively charged Lys residues that are involved in electrostatic binding to the SAM surface $[14,26]$.

The global coordinate system is positioned such that its origin coincides with the center of the sphere in its initial position (see Fig. 2). The positions of the point charges are given by vectors $\boldsymbol{r}_{i}=\left(x_{i}, y_{i}, z_{i}\right)$, with the respective coordinates $x_{i}, y_{i}, z_{i}$ determined from the crystal structure of Cyt-c (see Appendix A).

The redox site in the protein, taken to be the electron donor, is assumed to be located in the center of the heme and its coordinates are approximated by $\boldsymbol{r}_{D}=(0,-0.5 R, 0)$. Assuming a translational invariance in $(x, z)$ direction, the distance to the electrode (electron acceptor) is given by

$$
d=\left|y_{D}-y_{A}\right|=\left|y_{D}+d_{c}+R\right| .
$$

Here, $y_{D}$ and $y_{A}$ denote the position of the donator and the acceptor, respectively. To simulate the thermal motions of the immobilized Cyt-c, the protein model is allowed to rotate in the global coordinate system and to move along the $y$ axis without penetrating into the SAM. Upon rotation and translation of the sphere, the vectors of the point charges, $r_{i}$, and the donor, $r_{D}$, are thus transformed according to [27]

$$
\boldsymbol{r}_{i}^{\prime}=\underline{\underline{D}} \cdot \boldsymbol{r}_{i}+\boldsymbol{y}_{\text {trans }}, \quad \boldsymbol{r}_{D}^{\prime}=\underline{\underline{D}} \cdot \boldsymbol{r}_{D}+\boldsymbol{y}_{\text {trans }},
$$

where $D$ is a rotation matrix and $\boldsymbol{y}_{\text {trans }}=\left(0, y_{\text {trans }}, 0\right)$ a translation vector with the coordinate $y_{\text {trans }}$ ranging from 0 to $\infty$.

The rotation matrix $D$ is given in terms of the three Euler angles $(\alpha, \beta, \gamma)$, which along with the variable distance $y_{\text {trans }}$ represent the generalized coordinates for describing the dynamics of the system.

Due to the translational invariance in $(x, z)$ direction, only the $y$ coordinates of the point charges on the sphere and those of the heme center after rotation and translation are important. They can be obtained as [27]

$$
\begin{aligned}
y_{D / i}^{\prime}= & -x_{D / i}(\sin \gamma \cos \alpha-\sin \alpha \cos \beta \cos \gamma) \\
& +y_{D / i}(\cos \alpha \cos \beta \cos \gamma-\sin \alpha \sin \gamma) \\
& +z_{D / i}(\sin \beta \cos \gamma)+y_{\text {trans }} .
\end{aligned}
$$

\section{POTENTIAL ENERGY}

The potential energy of the model system is given by

$$
W=\sum_{i} q_{i} \phi\left(y_{i}^{\prime}\right),
$$

where $q_{i}=e_{0}$ are the four point charges on the sphere and $\phi\left(y_{i}^{\prime}\right)$ is the electrostatic potential at the position of each charge with $y_{i}^{\prime}$ given by Eq. (7). The surface charges on the electrode and the SAM, as well as the excess charges of the ions in the electrolyte solution are treated as the sources of the electrostatic potential $\phi$.

The electrostatic potential will be derived separately for the SAM and the electrolyte solution, as it is governed by different differential equations in the two spatial regions. The protein model is placed inside the external potential and assumed to represent only a weak perturbation of the overall potential $\phi$. It is therefore not considered for calculating the potential distribution.

(a) The $S A M$ can be treated as a dielectric region (dielectric constant $\varepsilon$ ) that is defined by the planes of the electrode and the SAM head groups, characterized by the charge densities $\sigma_{1}$ and $\sigma_{2}$, respectively [Fig. 2]. As there are no excess charges in the SAM, the potential distribution between the two surfaces is determined by Laplace's equation

$$
\frac{d^{2}}{d y^{2}} \phi(y)=0 \text {. }
$$

Two boundary conditions determine the solution of Eq. (9), namely, $\phi\left(-\left(d_{c}+R\right)\right)=\phi_{M}$ and $\phi(-R)=\phi_{c}$ with $\phi_{M}$ and $\phi_{c}$ denoting the potential at the electrode and the SAM surface, respectively. $\phi_{c}$ is a variable that depends on the electrode potential $\phi_{M}$, the SAM length and the electric field strength inside the SAM (see below). By integrating Eq. (9) twice and applying the boundary conditions, the potential distribution inside the SAM model is then given by

$$
\phi(y)=-\frac{\phi_{M}-\phi_{c}}{d_{c}}\left(y+d_{c}+R\right)+\phi_{M} .
$$

According to Eq. (10) the potential at the SAM surface is given by $\phi_{c}=\phi_{M}-E_{\mathrm{SAM}} d_{c}$.

The constant electric field strength $E_{\mathrm{SAM}}$ inside the SAM is obtained by calculating the force between a point charge and two charged surfaces with infinite dimensions according to

$$
E_{\mathrm{SAM}}=\frac{\sigma_{1}-\sigma_{2}}{2 \varepsilon_{0} \varepsilon}=\frac{\Delta \sigma}{2 \varepsilon_{0} \varepsilon},
$$

with $\Delta \sigma$ denoting the charge density difference between the electrode and the SAM surface.

(b) The potential in the electrolyte solution is caused by the ion distribution and can be described by the linearized Poisson-Boltzmann equation [28,29].

$$
\frac{d^{2}}{d y^{2}} \phi=\kappa^{2} \phi
$$

where $\kappa$ denotes the inverse Debye length of the solution. Equation (12) takes account of the screening of the potential by the mobile charges in the solution and the Debye length is the distance at which the modulus of the potential has dropped to $1 / e$ of its initial value.

The solution of Eq. (12) is given by 


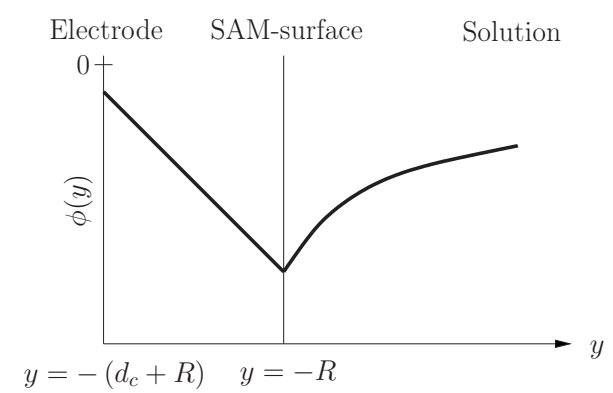

FIG. 3. Schematic presentation of the potential distribution in Fig. 4.

$$
\phi(y)=\left(\phi_{c}-\phi_{S}\right) e^{-\kappa(y+R)}+\phi_{S},
$$

where the integration constant $\phi_{S}$ denotes the potential in the solution for $y \rightarrow \infty$. Equation (13) fulfills the boundary condition $\phi(-R)=\phi_{c}$, such that it is consistent with Eq. (10) for the linear potential inside the SAM. The potential $\phi_{c}$ at the SAM surface is not constant but depends on the SAM length

$$
\phi_{c}=-\frac{\Delta \sigma}{2 \varepsilon_{0} \varepsilon} d_{c}+\phi_{M} .
$$

The complete electrostatic potential distribution in the SAM and the electrolyte solution is then given by

$$
\phi(y)=\left\{\begin{array}{ll}
-\frac{\Delta \sigma}{2 \varepsilon_{0} \varepsilon}\left(y+d_{c}+R\right)+\phi_{M}, & y \leq-R, \\
\widetilde{\phi}\left(d_{c}\right) e^{-\kappa(y+R)}+\phi_{S}, & y>-R,
\end{array},\right.
$$

with $\widetilde{\phi}\left(d_{c}\right):=\left(\phi_{M}-\phi_{S}-\frac{\Delta \sigma}{2 \varepsilon_{0} \varepsilon} d_{c}\right)$.

The evolution of the potential between electrode, SAM surface, and electrolyte solution is shown in Fig. 3. In Fig. 4, the potential distribution for different SAM lengths according to Eq. (14) is displayed. Here the charge density difference was varied according to experimental $\mathrm{p} K_{\mathrm{a}}$ values (see Appendix A).

Note that the coordinate system was shifted in Fig. 4 such that the position of the electrode surface coincides with the left boundary. The potential change varies from a linear to an exponential behavior at the SAM/electrolyte interface.

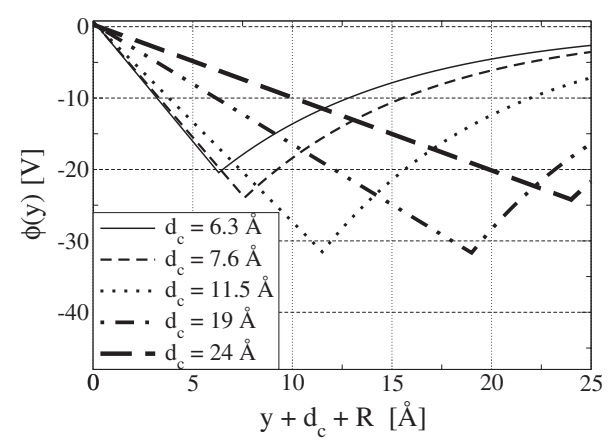

FIG. 4. Potential distribution for different SAM lengths. The coordinate system was shifted such that the position of the electrode surface coincides with the left boundary. For each $d_{c}$, the position of the SAM surface is indicated by the transition from the linear to the exponential region.
The electric field strength inside the SAM increases for decreasing SAM length, in agreement with the results of the electrostatic model described previously [16].

The slope of the potential in the region of the electrolyte solution is directly linked to the Coulomb force experienced by the protein. Since the electric field is given by the negative gradient of the potential, $E=-\nabla \phi$, a positive slope of the potential corresponds to a negative electric field and thus to an attractive Coulomb force on the positive charges on the model protein.

The magnitude of the slope depends on both the electric field strength inside the SAM and the lowest value of the potential at the SAM surface. Due to this, the electric field strength in the electrolyte solution first increases with decreasing SAM length but decreases again for short SAMs.

Experimental studies on the field-dependent conformational equilibria [16] and reorientation rate of Cyt-c [10] indicate that the electric field strength experienced by the immobilized protein steadily increases with decreasing SAM thickness. Thus, the calculated field strength in the SAM rather than that in the solution is correlated with the experimental data. In this sense, the present model does not fully account for the Coulombic forces acting on the immobilized protein located in the solution phase of the interface. This is due to unavoidable simplifications of the present model. Specifically, the charge distribution inside and on the surface of the protein are not considered on a molecular level and any perturbation of the interfacial potential distribution caused by the protein is neglected. In accordance with the experimental findings, one may thus take the electric field strength in the SAM as an approximate measure for the field experienced by the protein. Despite the simplification within our calculations, our model is able to account for the experimental observation of the saturation of the average electron transfer rate at short SAM lengths (see Sec. IV).

\section{AVERAGE ELECTRON TRANSFER RATE}

\section{A. Extraction of the electron transfer rate in the experiments}

Detailed experimental studies on the distance-dependence of the interfacial ET of Cyt-c have been carried out with time-resolved surface enhanced resonance Raman (SERR) spectroscopy $[2,4]$. In these experiments, the ET rate is not measured directly but is extracted from the time decay of the relative concentrations of the reduced or oxidized species of Cyt-c probed by SERR spectroscopy. The ratio of the relative concentration after a delay time $t$ and the relative concentration at zero delay time (at zero overpotential) is given by [2]

$$
\Gamma^{\exp }(t):=\frac{\Delta C(t)}{\Delta C(0)}=\exp \left(-2 k_{E T}^{0} t\right),
$$

where $k_{E T}^{0}:=k_{E T}(\eta=0)$ denotes the ET rate at zero overpotential. Taking into account the given time resolution and the accuracy of the concentration determination, the experimental data are, in most cases, satisfactorily described by a single exponential time decay, as given in Eq. (15) (vide infra). 


\section{B. Calculation of an average electron transfer rate}

In analogy to the experiments, we assume an exponential decay of the ET rate over time and calculate a statistical average of the quantity $\Gamma(t)=\exp \left(-2 k_{E T}^{0} t\right)$, in which $k_{E T}^{0}$ is substituted by the tunneling rate as given by Eqs. (2), (3), (5), and (7). The overpotential is set to $\eta=0$, according to the experimental studies on the distance-dependence of the ET rate [2].

According to Eq. (4), the statistical average of $\Gamma(t)$, in the framework of our model system, is given by

$\langle\Gamma(t)\rangle$

$$
=\frac{\int_{0}^{\infty} d\left(y_{\text {trans }}\right) \int_{0}^{2 \pi} d \alpha \int_{0}^{\pi} d \beta \int_{0}^{2 \pi} d \gamma \exp \left(-\frac{W}{k_{B} T}\right) \exp \left(-2 k_{E T}^{0} t\right)}{\int_{0}^{\infty} d\left(y_{\text {trans }}\right) \int_{0}^{2 \pi} d \alpha \int_{0}^{\pi} d \beta \int_{0}^{2 \pi} d \gamma \exp \left(-\frac{W}{k_{B} T}\right)},
$$

where Eqs. (7) and (8) must be substituted for the potential energy term $W$.

We then obtain the average ET rate by calculating the slope of the function $g(t)=\ln \langle\Gamma(t)\rangle$ at delay time $t$

$$
\left\langle k_{E T}^{0}\right\rangle(t)=-\frac{1}{2} \lim _{\Delta t \rightarrow 0} \frac{g(t+\Delta t)-g(t-\Delta t)}{2 \Delta t} \quad(t>0) .
$$

Provided that $\langle\Gamma(t)\rangle$ is a monoexponential function of $t$, as is $\Gamma(t)$, the average ET rate obtained with Eq. (17) is independent of the inserted delay time $t$. As will be shown below, this is not the case for all $t$. Note that this approach is not equivalent to averaging over the electron tunneling rate, which in turn is not suitable for a comparison with the experimental data (see Appendix C).

\section{Numerical results}

Equation (16) was evaluated numerically using a fourthorder Simpson rule. The upper integration boundary of the integral over $y_{\text {trans }}$ was set to $300 \AA$. For this value, the results converged sufficiently. Yet, for the experimentally relevant region of SAM lengths ( $\left.5 \AA \leq d_{c} \leq 25 \AA\right)$, the results converged even at a much smaller value of $20 \AA$, which is also sufficiently large to cover the relevant volume of molecules investigated in the SERR experiments. Only for $d_{c}$ $<1 \AA$, the results converged much more slowly (see below). The delay time was set to $t=10 \mathrm{~ms}$. Results calculated for the average ET rate constant according to Eq. (17) are shown in Fig. 5.

The calculations nicely reproduce the plateau region between 15 and $5 \AA$ in the dependence of the average electron transfer constant on the SAM length $d_{c}$ [2], although protein reorientation has been neglected. This region results from the SAM length dependent electric field strength, which affects the orientation probabilities of the protein (see Appendix B). They first decrease with growing SAM lengths $d_{c} \geq 0.5 \AA$ and then again increase for thick SAMs, which can be attributed to the potential distribution at different SAM lengths [Fig. 4]. With decreasing $d_{c}$, the field strength in the SAM

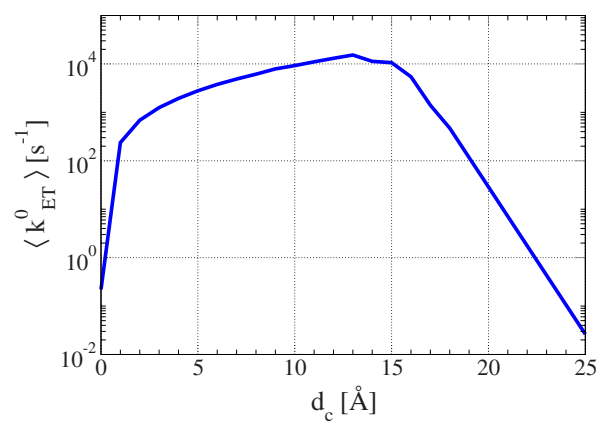

FIG. 5. (Color online) Calculated average ET rate constant as a function of the SAM length, at a delay time of $10 \mathrm{~ms}$.

increases, which may be taken as a measure for the increasing field strength experienced by the protein (see above) [30].

The calculations further predict a decrease in the average ET rate constant at distances shorter than $6 \AA$. Although such short distances cannot be studied experimentally using SAM-coated electrodes, we may compare this prediction with experimental results obtained for Cyt-c adsorbed on electrodes coated with sulfate and phosphonate-terminated alkanethiols [4]. For both systems, the high charge densities that are due to the very low $\mathrm{p} K_{\mathrm{a}}$ values of the SAM tail groups lead to electric field strengths at the Cyt-c binding site that are distinctly higher than those for a SAM built up by the shortest possible carboxyl-terminated alkanethiol $\left(d_{c}\right.$ $=6.3 \AA$ ). In fact, the experimentally determined ET rate constants are distinctly smaller than the plateau value, thereby confirming the predictions of the present theoretical model.

\section{Influence of the delay time}

In time-resolved SERR experiments, two different processes can be probed separately following a rapid potential jump. Reduction or oxidation of the heme (ET) is monitored on the basis of the characteristic oxidation marker bands of the heme, and the changes of the orientational distribution of the oxidized Cyt-c are probed via the intensity changes of the porphyrin modes of different symmetry [10]. At SAMs of long mercaptonalkane chains, the ET rate is found to be slower than the orientational changes, implying that electron tunneling is the rate-limiting step for the overall redox process. With decreasing chain length, the electron tunneling rate becomes faster, whereas, due to the increasing electric field strength, the orientational changes are slowed down until the reorientation rate constant becomes rate-limiting in the "plateau" region. In this regime, the changes of the orientational distribution probed in the SERR experiments may reflect the transition between Cyt-c molecules of different orientations and the successive reduction in Cyt-c molecules according to their orientation-dependent electron tunneling probabilities [14]. Both effects are expected to cause different deviations from a monoexponential behavior of the ET rate when a large dynamic range is considered.

Within the present model, which accounts for the orientational distribution but not for the orientational dynamics, we have, therefore, investigated the deviations from the mo- 


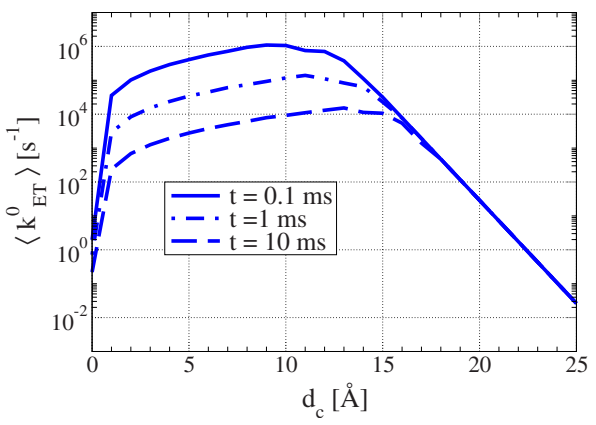

FIG. 6. (Color online) Average ET rate calculated as a function of the SAM length for different delay times $t$ according to Eq. (17)

noexponential behavior by calculating the average ET rate constant for different delay times $t$ in Eq. (17), corresponding to different sampling times in the time-resolved SERR experiments. The results, shown in Fig. 6, reveal that the characteristic "saturation" behavior of the ET rate constant for short SAM lengths is reached for different delay times.

Upon setting the delay time to smaller values, the onset of the saturation occurs for shorter SAM lengths. In the limit of $t \rightarrow 0$, the plateau vanishes completely and the average rate constant approaches the tunneling rate limit even for the shortest SAM lengths. However, for long SAM lengths (19 $\left.\AA \leq d_{c} \leq 25 \AA\right)$, a deviation from the exponential increase in the ET rate, and thus a deviation from the electron tunneling regime, only occurs for long delay times, whereas for short SAMs the ET rate reacts much more sensitively to the delay time used in the calculations. In the limiting case of $t \rightarrow \infty$, the plateau covers the whole range from $d_{c}=0$ to $25 \AA$ and the ET rate approaches zero. The distancedependent saturation of the ET rate only occurs in a range of delay times where the averaged function $\langle\Gamma(t)\rangle$ is not an exponential function of $t$. For each SAM length there is a range $\left(0, t_{0}\right)$ of delay times, starting at $t=0$, where $g(t)$ $=\ln \langle\Gamma(t)\rangle$ is a linear function with a constant slope, i.e., $\langle\Gamma(t)\rangle$ is an exponential function in that range. For values $t$ $>t_{0}$, the modulus of the slope of $g(t)$ decreases, which in turn leads to a decreasing ET rate. This is shown in Fig. 7, upon plotting the function $g(t)$ versus the delay time for SAM lengths of $d_{c}=6.3,7.6$, and $11.5 \AA$.

The shorter the SAM length, the smaller is the interval $\left(0, t_{0}\right)$ where $\langle\Gamma(t)\rangle$ is a monoexponential function of $t$ (see Table I). In these intervals, the average rate is of the same order of magnitude as the tunneling rate, so the saturation is obtained only for a delay time outside the interval $\left(0, t_{0}\right)$.

Upon comparing these results with the experimental data, one has to recall that time-resolved SERR spectroscopic measurements refer to a time window of not more than two decades, covering ca. $90 \%$ of the maximum concentration change. For thick SAMs with $d_{c}=24.0$ and $19.0 \AA$, the experimental time windows fall into the respective intervals for which the model predicts a monoexponential behavior, in agreement with the experiments. For shorter SAMs, the predicted exponential intervals are not included in the sampling window of the SERR measurements. Thus, fast ET rates for a minor fraction of Cyt-c molecules (corresponding to the exponential range according to Table I) may well occur. Its
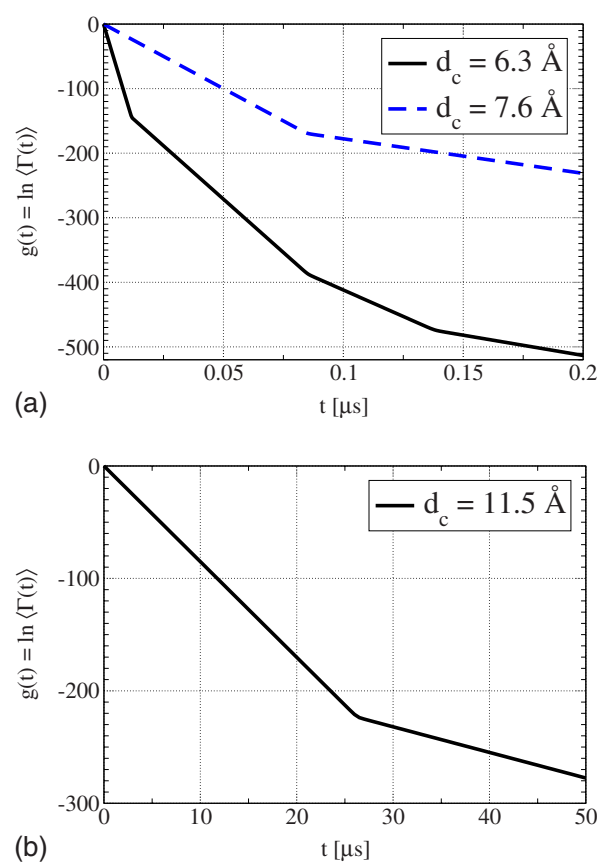

FIG. 7. (Color online) Logarithm of the calculated averaged quantity $\langle\Gamma(t)\rangle$ for $d_{c}=6.5,7.6$ and $11.5 \AA$ as a function of the delay time $t$ in Eq. (17)

detection requires the expansion of the dynamic range in future time-resolved SERR experiments. Beyond this time interval $\left(0, t_{0}\right)$, the average ET rate follows a complex function. The fact that, in previous SERR experiments, this region could be satisfactorily approximated by a monoexponential decay may imply, on the one hand, that the error margins of the experimental data did not justify simulations by more complex (multiexponential) functions. On the other hand, in this time range the dynamics of protein reorientation are most likely rate-determining for the reduction/oxidation of the main fraction of Cyt-c, which may result in a nearly monoexponential behavior of the overall ET rate.

It is interesting to note that a clearly nonexponential behavior has been observed in time-resolved SERR experiments on the oxidation of the Cyt-c on $\mathrm{C}_{11}$-coated electrodes $\left(d_{c}=19.0 \AA\right)$ when the size of the potential jump was increased from 40 to $125 \mathrm{mV}$. The resulting decay curve was similar to that in Fig. 7 [31] and revealed an additional slower phase (ca. $16 \mathrm{~s}^{-1}$ ) compared to the monoexponential kinetics $\left(42 \mathrm{~s}^{-1}\right)$ for the oxidation using smaller potential jumps. This behavior was attributed to the potentialdependent orientational distribution of the immobilized Cyt-c, implying that the reorientation rate of ferrous Cyt-c, at least at more negative potentials, is distinctly slower $\left(\leq 16 \mathrm{~s}^{-1}\right)$ than that of ferric Cyt-c (ca. $\left.380 \mathrm{~s}^{-1}\right)$.

TABLE I. Time Ranges $\left(0, t_{0}\right)$ where $\langle\Gamma(t)\rangle$ is a monoexponential function, for different SAM lengths.

\begin{tabular}{lccc}
\hline \hline $\begin{array}{l}d_{c} \\
(\AA)\end{array}$ & 6.3 & 7.6 & 11.5 \\
\hline$t_{0}$ & $0.015 \mu \mathrm{s}$ & $0.08 \mu \mathrm{s}$ & $25 \mu \mathrm{s}$ \\
\hline \hline
\end{tabular}




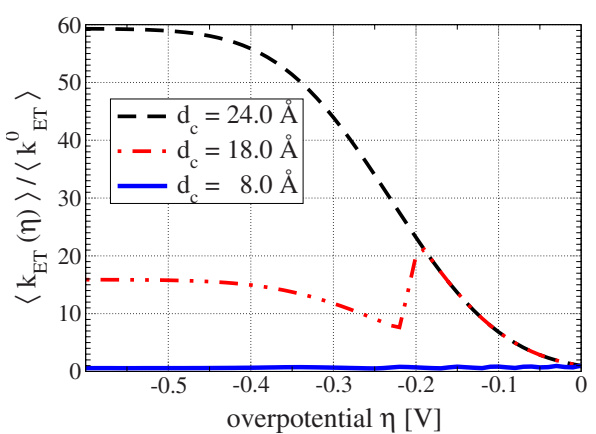

FIG. 8. (Color online) Overpotential dependence of the average ET rate for different SAM lengths; Delay time: $t=10 \mathrm{~ms}$

\section{OVERPOTENTIAL DEPENDENCE OF THE ELECTRON TRANSFER RATE}

In this section we analyze the overpotential-dependence of the ET rate (see Eq. (2)) to further examine the interplay between the electron tunneling and protein reorientation regime. In Fig. 8, the ratio $\left\langle k_{E T}(\eta)\right\rangle /\left\langle k_{E T}^{0}\right\rangle$ of the rate at overpotential $\eta$ to the rate at zero overpotential is shown versus a negative overpotential for three different SAM lengths. The rate was calculated with Eqs. (16) and (17) by averaging over an exponential function of the tunneling rate. The delay time was again set to $t=10 \mathrm{~ms}$.

For $d_{c}=24 \AA$, which refers to the long-range electron tunneling regime, the overpotential dependence of the average rate and the tunneling rate are equal, whereas for short SAMs, the ratio of the average rates remains virtually constant, reflecting the regime, where the proteins orientational distribution is crucial. A transition between these two regimes can be seen for a SAM length of $d_{c}=18.0 \AA$, which is roughly the value at which the onset of the rate saturation occurs in Fig. 5. These findings nicely reproduce the experimental $[2,24]$ data and further confirm the conclusion that a process other than electron tunneling is the rate-limiting step for short SAM lengths.

\section{CONCLUSION}

The present model provides a qualitative description of the unusual distance-dependence of the ET processes of Cyt-c on SAM-coated electrodes. It takes into account the electric field dependent distribution of protein orientations, which leads to a distribution of effective donor-acceptor distances and thus to a distribution of electron tunneling rates. The model is not based on the molecular description of the system and thus can easily be extended to other proteins and interfaces. Conversely, it does not provide information about the mechanism and dynamics of the interfacial process on a molecular level. However, as a stationary model it can account for the saturation of the ET rate as observed in experimental studies [2]. The calculations show that the protein orientational distribution plays a crucial role for the average ET rate and thereby supports the experimental observations that electric field effects, which ultimately cause the different orientation probabilities for different SAM lengths, directly influence the electron transfer dynamics of protein systems.
To further check the model and to assess the effect of protein reorientation dynamics that are not included in the model, further time-resolved SERR experiments should cover a larger time range. The model may thus serve as a starting point for a comprehensive description of the interfacial ET process of Cyt-c and of redox proteins in general.

\section{ACKNOWLEDGMENTS}

This work was supported by the DFG Cluster of Excellence UniCat and the Fonds der Chemischen Industrie.

\section{APPENDIX A: PARAMETERS FOR NUMERICAL CALCULATIONS}

The model system is constituted by horse heart Cyt-c (PDB code: 1HRC) adsorbed on a SAM-coated silver electrode.

Cyt-c is approximated by a sphere with a radius $R$ $=13 \AA$. This value is the radius of gyration of Cyt-c, which was used in molecular simulation studies [26].

The dielectric constant of the SAM is assumed to be $\varepsilon_{1}$ $=2.26[16]$.

The computations were done for an inverse Debye length of $\kappa=1.1 \cdot 10^{9} \mathrm{~m}^{-1}$, corresponding to the experimental conditions for the SERR studies to which the present results are compared [2]. The reorganization energy was taken as $\lambda$ $=0.23 \mathrm{eV}$, as determined for Cyt-c adsorbed on a $\mathrm{C}_{15}$-SAM-coated electrode [24]. The constant density of states for the silver electrode was set to $\rho=1 \mathrm{eV}^{-1}$, which is on the same order of magnitude as the energy-dependent total density of states as given in [32] and thus constitutes a good estimate.

\section{Positions of point charges}

The positions of the four point charges responsible for the electrostatic binding were estimated in analogy to four cationic Lys side chains of the Cyt-c (crystal structure data 1HRC) using the program VMD [11]. The coordinates are

$$
\begin{gathered}
\left(\text { Lys 25): } \boldsymbol{r}_{1} \approx(0,-0.7,1) R\right. \\
\left(\text { Lys 27): } \boldsymbol{r}_{2} \approx(0,-0.7,0.7) R\right. \\
\left(\text { Lys 72): } \boldsymbol{r}_{3} \approx(0,-0.7,-0.7) R\right. \\
\left(\text { Lys 79): } \boldsymbol{r}_{4} \approx(0.7,-0.5,0) R\right.
\end{gathered}
$$

Note that the exact positions of these point charges has no impact on the calculations; moreover, it is not intended to precisely mimic the charge distribution of Cyt-c in this respect.

\section{Potential drop between electrode and solution}

The interfacial potential distribution is described in analogy to previous models [33] with $\phi_{M}$ and $\phi_{S}$ denoting the potentials on the electrode and of the solution, respectively. The potential drop between electrode and solution is given 
TABLE II. Redox potentials of Cyt-c adsorbed on SAM-coated Ag-electrodes (Ref. [16]).

\begin{tabular}{lcc}
\hline \hline SAM & $\begin{array}{c}d_{c} \\
(\AA)\end{array}$ & $\begin{array}{c}\phi_{\text {redox }} \\
(\mathrm{mV})\end{array}$ \\
\hline $\mathrm{C}_{2}$ & 6.3 & 2.5 \\
$\mathrm{C}_{3}$ & 7.6 & -9.6 \\
$\mathrm{C}_{6}$ & 11.5 & -14.2 \\
$\mathrm{C}_{11}$ & 19 & -31.0 \\
$\mathrm{C}_{16}$ & 24 & -39.0 \\
\hline \hline
\end{tabular}

by $\phi_{M}-\phi_{S}=\phi_{\mathrm{appl}}-\phi_{\mathrm{pzc}}$, where $\phi_{\mathrm{appl}}$ is the applied electrode potential and $\phi_{\mathrm{pzc}}$ denotes the potential of zero charge of the electrode. Equation (14) can then be rewritten as

$$
\phi(y)= \begin{cases}-\frac{\Delta \sigma}{2 \varepsilon_{0} \varepsilon}\left(y+d_{c}+R\right)+\phi_{\mathrm{appl}}-\phi_{\mathrm{pzc}}+\phi_{S}, & y \leq-R \\ \widetilde{\phi}\left(d_{c}\right) e^{-\kappa(y+R)}+\phi_{S}, & y>-R\end{cases}
$$

with $\widetilde{\phi}\left(d_{c}\right)=\left(\phi_{\mathrm{appl}}-\phi_{\mathrm{pzc}}-\frac{\Delta \sigma}{2 \varepsilon_{0} \varepsilon} d_{c}\right)$. The constant potential $\phi_{S}$ in the solution can be chosen as an arbitrary value, as it cancels out in the averaging integral (16).

In this paper, we calculate an average ET rate for a model system for Cyt-c on SAM-coated Ag electrodes. Therefore, the parameters in Eq. (A1) for the potential are taken from the experiments, as far as they are known. The potential of zero charge varies with SAM length and is taken from a linear fit to the following experimental values: $\phi_{\mathrm{pzc}}^{\mathrm{Ag}}\left(d_{c}=0\right)$ $=-0.97 \mathrm{~V}[34], \phi_{\mathrm{pzc}}^{\mathrm{Ag} / \mathrm{SAM}}\left(d_{c}=19.0 \AA\right)=-0.45 \mathrm{~V}[35]$.

The distance-dependence of the ET rate is measured at zero overpotential, that is, the applied potential is set equal to the apparent redox potential of the adsorbed Cyt-c, which depends on the SAM length, as well. For the numerical calculations, the applied potential is obtained as an exponential fit to the experimental values of the redox potential (Table II).

The charge density $\sigma_{2}$ on the SAM-surface is estimated using a relation from [16]

$$
\mathrm{p} K_{\mathrm{a}}=\mathrm{pH}-\log \left(\frac{\sigma_{2}}{\sigma_{2}^{\max }-\sigma_{2}}\right),
$$

where $\sigma_{2}^{\max }=-0.816 \mathrm{C} \mathrm{m}^{-2}$ is the maximum value in the case when all carboxylate groups of the SAM carry a negative charge. The $\mathrm{p} K_{\mathrm{a}}$ depends on the SAM length. Values were measured for SAMs on an Au electrode in [36] (see Table III), where it was found that the $\mathrm{p} K_{\mathrm{a}}$ increases with increasing SAM lengths. The absolute values for SAMs on Ag electrodes were not available, yet the general trend can be assumed to be the same as for gold electrodes. Thus, the values measured in [36] were taken as a first estimate, leading to an approximately linear $d_{c}$-dependence of the $\mathrm{p} K_{\mathrm{a}}$ values. The charge density $\sigma_{2}$ can then be obtained as
TABLE III. Measured $\mathrm{p} K_{\mathrm{a}}$ values for SAMs on gold electrodes (Ref. [36]).

\begin{tabular}{lcc}
\hline \hline $\mathrm{SAM}$ & $\begin{array}{c}d_{c} \\
(\AA)\end{array}$ & $\mathrm{p} K_{\mathrm{a}}$ \\
\hline $\mathrm{C}_{2}$ & 6.3 & 6.1 \\
$\mathrm{C}_{3}$ & 7.6 & 5.3 \\
$\mathrm{C}_{11}$ & 19.0 & 7.3 \\
$\mathrm{C}_{16}$ & 24.0 & 7.9 \\
\hline \hline
\end{tabular}

$$
\sigma_{2}\left(d_{c}\right)=\frac{\sigma_{2}^{\max }}{1+\exp \left(\mathrm{p} K_{\mathrm{a}}\left(d_{c}\right)-\mathrm{pH}\right)} .
$$

For the charge density $\sigma_{1}$ on the electrode, one can assume $\sigma_{1} \approx-\sigma_{2}$, in the case of Cyt-c directly adsorbed to an electrode or to an anionic layer [37]. For SAM-coated electrodes, the relation will certainly be different, yet can be used to obtain a reasonable estimate for $\sigma_{1}$, and thereby for $\Delta \sigma$. The choice of $\Delta \sigma$ (i.e., $\sigma_{1}$ and $\sigma_{2}$ ) is in fact critical for the results, for instance, of the magnitude of the average electron tunneling rate (cf. Figure 12), although it does not significantly affect the qualitative description.

Defining $q:=\left|\boldsymbol{r}-\boldsymbol{r}_{D}\right|, q^{\prime}:=\left|\boldsymbol{r}-\boldsymbol{r}_{A}\right|$ and the donor-acceptor distance $d:=\left|\boldsymbol{r}_{D}-\boldsymbol{r}_{A}\right|$, the three coordinates are [38]: $\mu=\frac{q+q^{\prime}}{d}$, $\mu \in[1, \infty), \nu=\frac{q-q^{\prime}}{d}, \nu \in[-1,1]$, and $s \in[0,2 \pi]$, where $\mathrm{s}$ is the azimuthal angle around the $\left(\boldsymbol{r}_{D}, \boldsymbol{r}_{A}\right)$ axis.

The volume element for this choice of coordinates is $d V$ $=\frac{1}{8} d^{3}\left(\mu^{2}-\nu^{2}\right) d \mu d \nu d s$.

\section{APPENDIX B: PROBABILITY DENSITIES}

The probability density $f$ of the variable $y$-distance $y_{\text {trans }}$ is plotted in Fig. 9 for different SAM lengths. Here, the three angles were set to fixed values of $\alpha=\beta=\gamma=0$ in Eq. (7), such that the probability density can simply be calculated as

$$
f\left(y_{\text {trans }}\right)=\frac{\exp \left(-\frac{W}{k_{B} T}\right)}{\int_{0}^{\infty} d\left(y_{\text {trans }}\right) \exp \left(-\frac{W}{k_{B} T}\right)} .
$$

Figure 9(b)) reveals that there is a transition between $d_{c}$ $=0.1 \AA$ and $d_{c}=0.5 \AA$. For $d_{c}=0.1 \AA$, it is more probable for the model protein to be further away from the SAM. This is the reason why the results for the average ET rate converge much more slowly when the maximum value for the variable distance $y_{\text {trans }}$ is increased, thereby causing a sharp drop of the ET rate below $d_{c}=1 \AA$ in Fig. 5. The transition of the probability density in Fig. 9(b)) is due to the change in sign of the Coulomb force (from attractive to repulsive) for very small values of $d_{c}$. This is illustrated in Fig. 10, where the potential distribution is plotted as in Fig. 4, but for smaller values of $d_{c}$. The negative slope of the potential in the electrolyte solution for $d_{c}=0.1 \AA$ corresponds to a positive electric field and thus to a repulsive Coulomb force on the positive charges on the model protein, whereas for $d_{c}$ $=0.5 \AA$ the slope of the potential is positive, which corre- 



FIG. 9. Probability density with respect to the variable $y$-distance $y_{\text {trans }}$ for different SAM lengths.

sponds to a negative electric field and an attractive Coulomb force on the model protein.

For a fixed orientation, the electron tunneling rate increases exponentially without any saturation. This is depicted in Fig. 11.

\section{APPENDIX C: AVERAGING OVER THE TUNNELLING RATE}

Here an average ET rate is calculated by averaging over the tunneling rate directly, rather than over an exponential function as in Eq. (16). This procedure filters out the influence of protein reorientation on the electron transfer independently of additional effects of the experimental conditions. The results further illuminate the effect of the exponential function in the averaging integral Eq. (16).

We calculate an average ET rate as follows:

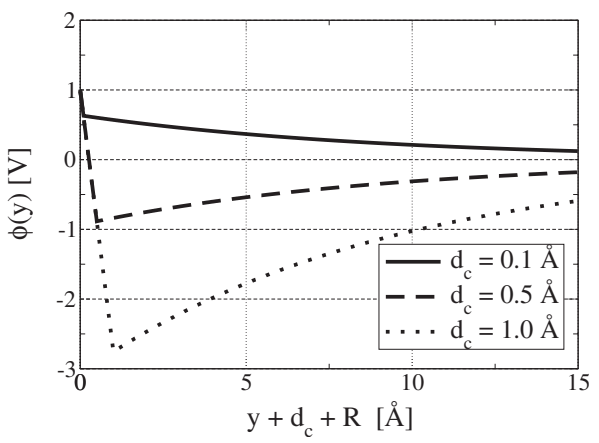

FIG. 10. Potential distribution for short SAM lengths



FIG. 11. (Color online) Black (upper) line: ET rate for a fixed molecule (tunneling rate); when all reorientation is switched off; Blue (dashed) line: Ensemble average of the ET rate for, averaged over the orientational distribution with Eq. (16)

$$
\left\langle k_{E T}^{0}\right\rangle=\frac{\int_{0}^{\infty} d\left(y_{\text {trans }}\right) \int_{0}^{2 \pi} d \alpha \int_{0}^{\pi} d \beta \int_{0}^{2 \pi} d \gamma \exp \left(-\frac{W}{k_{B} T}\right) k_{E T}^{0}}{\int_{0}^{\infty} d\left(y_{\text {trans }}\right) \int_{0}^{2 \pi} d \alpha \int_{0}^{\pi} d \beta \int_{0}^{2 \pi} d \gamma \exp \left(-\frac{W}{k_{B} T}\right)},
$$

where again the tunneling rate as given by Eqs. (2), (3), and (7), at overpotential $\eta=0$ is substituted for $k_{E T}^{0}$. The numerical results for different charge densities are shown in Fig. 12. Here, the maximum distance in $y$ direction was again set to $300 \AA$.

The charge density difference $\Delta \sigma$ for the full blue curve in Fig. 12 was varied according to experimental $\mathrm{p} K_{\mathrm{a}}$ values, as for the full blue curve in Fig. 5. It is obvious that a saturation of the ET rate as in Fig. 5 does not occur for the actual rate. Rather, the rate behaves as the tunneling rate and increases exponentially for decreasing SAM lengths. Only at a very short SAM length does the rate reach a maximum and falls off rapidly for further decreasing values of $d_{c}$. This effect is due to a repulsive Coulomb force on the model protein and is the same mechanism that causes the sharp decrease in the ET rate for very short SAM lengths in Fig. 5.

To further illustrate this effect, the average rate is also plotted for two fixed values of the charge density difference (red dashed-dotted and green dashed curve in Fig. 12). In these cases, the critical SAM length, where the Coulomb

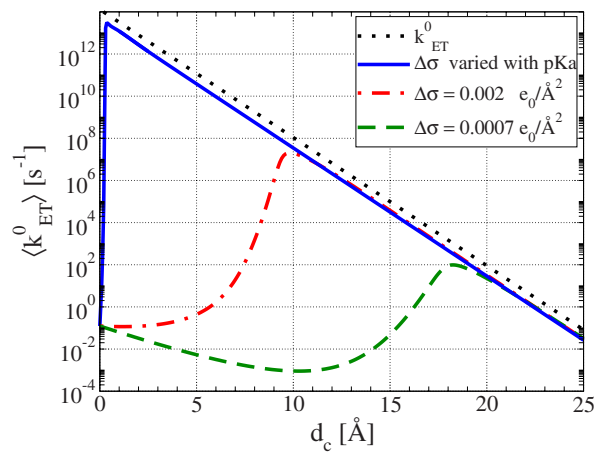

FIG. 12. (Color online) Average of the electron tunneling rate [obtained with Eq. (C1)] for different charge densities 
force changes from attractive to repulsive, and thereby the point where the kink in the ET rate occurs, is larger than for the more realistic values of $\Delta \sigma$ that vary with the SAM length.
[1] A. Avila, B. W. Gregory, K. Niki, and T. M. Cotton, J. Phys. Chem. B 104, 2759 (2000).

[2] D. H. Murgida and P. Hildebrandt, J. Am. Chem. Soc. 123, 4062 (2001).

[3] Q. Chi, J. Zhang, J. E. T. Andersen, and J. Ulstrup, J. Phys. Chem. B 105, 4669 (2001).

[4] D. H. Murgida and P. Hildebrandt, Phys. Chem. Chem. Phys. 7, 3773 (2005).

[5] J. Wei, H. Liu, D. E. Khoshtariya, H. Yamamoto, A. Dick, and D. H. Waldeck, Angew. Chem., Int. Ed. 41, 4700 (2002).

[6] J. J. Wei, H. Liu, K. Niki, E. Margoliash, and D. H. Waldeck, J. Phys. Chem. B 108, 16912 (2004).

[7] D. E. Khoshtariya, J. Wei, H. Liu, H. Yue, and D. H. Waldeck, J. Am. Chem. Soc. 125, 7704 (2003).

[8] Z. Q. Feng, S. Imabayashi, T. Kakiuchi, and K. Niki, J. Electroanal. Chem. 394, 149 (1995).

[9] Z. Q. Feng, S. Imabayashi, T. Kakiuchi, and K. Niki, J. Chem. Soc., Faraday Trans. 93, 1367 (1997).

[10] A. Kranich, H. K. Ly, P. Hildebrandt, and D. H. Murgida, J. Am. Chem. Soc. 130, 9844 (2008).

[11] W. Humphrey, A. Dalke, and K. Schulten, J. Mol. Graphics 14, 33 (1996).

[12] P. Zuo, T. Albrecht, P. D. Baker, D. H. Murgida, and P. Hildebrandt, Phys. Chem. Chem. Phys. 11, 7430 (2009).

[13] D. L. Brautigan, S. Ferguson-Miller, and E. Margoliash, Methods Enzymol. 53, 128 (1978).

[14] D. A. Paggi, D. F. Martín, A. Kranich, P. Hildebrandt, M. Martí, and D. H. Murgida, Electrochim. Acta 54, 4963 (2009).

[15] R. Pincak and M. Pudlak, Phys. Rev. E 64, 031906 (2001).

[16] D. H. Murgida and P. Hildebrandt, J. Phys. Chem. B 105, 1578 (2001).

[17] The overpotential $\eta$ is the difference between the applied electrode potential and the redox potential of the system: $\eta$ $=\phi_{\text {appl }}-\phi_{\text {redox }}$.

[18] R. A. Marcus and N. Sutin, Biochim. Biophys. Acta 811, 265 (1985).

[19] T. Renger and R. A. Marcus, J. Phys. Chem. A 107, 8404 (2003).

[20] R. A. Marcus, J. Chem. Phys. 43, 679 (1965).
[21] H. Yue, D. Khoshtariya, D. H. Waldeck, J. Grochol, P. Hildebrandt, and D. H. Murgida, J. Phys. Chem. B 110, 19906 (2006).

[22] M. D. Newton and N. Sutin, Annu. Rev. Phys. Chem. 35, 437 (1984).

[23] D. H. Murgida and P. Hildebrandt, Acc. Chem. Res. 37, 854 (2004)

[24] D. H. Murgida and P. Hildebrandt, J. Phys. Chem. B 106, 12814 (2002).

[25] L. D. Landau and E. M. Lifshitz, Statistical Physics (Butterworth-Heinemann, London, 1980).

[26] J. Zhou, J. Zheng, and S. Jiang, J. Phys. Chem. B 108, 17418 (2004).

[27] H. Goldstein, Classical Mechanics (Addison-Wesley Publishing, Reading, MA,1980).

[28] J. Stahlberg, U. Appelgren, and B. Jönsson, J. Colloid Interface Sci. 176, 397 (1995).

[29] B. Brocklehurst, J. Phys. Chem. 83, 536 (1979).

[30] Only for very short subatomic distances $(<1 \AA)$, the electric field changes the sign and repulsive interactions prevail (see Appendix B). This region, however, is not relevant for the analysis of the ET process.

[31] D. H. Murgida and P. Hildebrandt, Angew. Chem., Int. Ed. 40, 728 (2001).

[32] D. A. Papaconstantopoulos, Handbook of the Band Structure of Elemental Solids (Plenum Press, New York, 1986).

[33] S. Lecomte, P. Hildebrandt, and T. Soulimane, J. Phys. Chem. B 103, 10053 (1999).

[34] G. Valette and A. Hamelin, J. Electroanal. Chem. Interfacial Electrochem. 45, 301 (1973).

[35] J.-J. Feng, D. H. Murgida, U. Kuhlmann, T. Utesch, M. A. Mroginski, P. Hildebrandt, and I. M. Weidinger, J. Phys. Chem. B 112, 15202 (2008).

[36] Z. Dai and H. Ju, Phys. Chem. Chem. Phys. 3, 3769 (2001).

[37] F. Siebert and P. Hildebrandt, Vibrational Spectroscopy in Life Science (Wiley-VCH, Berlin, 2008).

[38] P. Atkins and R. Friedman, Molecular Quantum Mechanics (Oxford University Press, New York, 2005). 